\title{
Optical-gradient antireflective coatings for 157-nm optical lithography applications
}

\author{
Hsuen-Li Chen, Wonder Fan, Tzyy-Jyann Wang, Fu-Hsiung Ko, Run-Sheng Zhai, \\ Chien-Kui Hsu, and Tung-Jung Chuang
}

\begin{abstract}
We demonstrate an optical-gradient bottom antireflective coating (BARC) film, which can be easily prepared by treating a silicon nitride film with oxygen plasma. The oxygen composition is gradually decreased inside the silicon nitride film. The optical constants of the silicon nitride film are also changed gradually. A reflectance of less than $1 \%$ for various highly reflective substrates with high thicknesscontrolled tolerance has been achieved. The optical-gradient film is also shown to have high thermal stability during the postexposure bake procedure. Results indicate that the optical-gradient-type BARC is suitable in both $\mathrm{ArF}$ and $\mathrm{F}_{2}$ excimer lasers for sub-70-nm lithography applications. (C) 2004 Optical Society of America

OCIS codes: $\quad 310.1210,310.1860,310.3840,220.3740$.
\end{abstract}

\section{Introduction}

In the 2001 International Technology Roadmap for Semiconductors $^{1}$ (ITRS), ArF (193-nm) and $\mathrm{F}_{2}$ (157$\mathrm{nm}$ ) excimer-laser-based lithography combined with resolution-enhancement techniques would lead integrated circuit technologies to the generation of $90-$ and 65-nm nodes, respectively. It is therefore important to find a high-performance bottom antireflective coating (BARC) that works in this spectral regime.

The concept behind using a multilayer BARC is that the extinction coefficient $(k)$ of each BARC layer is gradually increased layer by layer and is the highest at the bottom. ${ }^{2,3}$ The combined multilayer thinfilm stack creates a gradual change of optical constants to minimize the reflectance at each BARC layer interface. The light reflected from a highly reflective substrate can be absorbed layer by layer.

H.-L. Chen (hlchen@ndl.gov.tw) is with the Department of Material Science and Engineering, National Taiwan University, Taipei, Taiwan. W. Fan and F.-H. Ko are with the National Nano Device Laboratory, 1001-1 Ta Hsueh Road, Hsinchu, Taiwan. T.-J. Wang is with the Institute of Electro-Optical Engineering, National Taipei University of Technology, Taipei, Taiwan. R.-S. Zhai, C.-K. Hsu, and T.-J. Chuang are with the Center for Condensed Matter Sciences, National Taiwan University, Taipei, Taiwan.

Received 27 June 2003; revised manuscript received 11 November 2003; accepted 30 December 2003.

0003-6935/04/102141-05\$15.00/0

(C) 2004 Optical Society of America
Thus a graded-absorption multilayer BARC can be used in various reflective substrates with high thickness-controlled tolerance. However, the required optical constants and thicknesses of each layer in the multilayer structure should be tuned carefully by varying the gas-flow-rate ratio of reactive gases in a chemical vapor deposition process. Nitride-based (silicon nitride, silicon oxynitride) films have been demonstrated to have suitable optical characteristics for $\mathrm{KrF}$ and $\mathrm{ArF}$ lithography BARC applications., ${ }^{4,5}$ Recently, hexamethyldisiloxane and carbon films have been shown to be suitable as BARC layers for $\mathrm{F}_{2}$ lithography. ${ }_{6,7}$ However, nitride-based BARC films are also preferred in $\mathrm{F}_{2}$ lithography.

Here we demonstrate an optical-gradient-type BARC structure for both $\mathrm{ArF}$ and $\mathrm{F}_{2}$ lithography. Figure 1 shows an optical-gradient silicon nitride film deposited by conventional plasma-enhanced chemical vapor deposition and then treated with oxygen plasma. The surface of the silicon nitride film is oxidized after the plasma treatment. The composition and depth profile of the optical-gradient film was measured by x-ray photoelectron spectroscopy and secondary ion mass spectroscopy. The oxygen composition is gradually decreased inside the silicon nitride film. The optical constants of the film are also found to change gradually by simulation with the effective medium model. ${ }^{8}$ The high oxygen composition of the film is accompanied by low absorption in the deep-ultraviolet and vacuum-ultraviolet regimes. Therefore optical-gradient-type antireflective coat- 


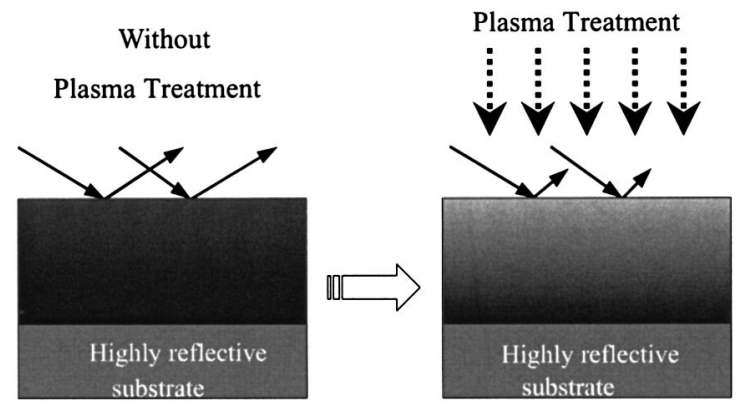

Fig. 1. Schematic diagram of an optical-gradient-type antireflective coating, which is formed by plasma treatment.

ings can be fabricated easily by use of the oxygen plasma treatment.

Chemically amplified resists with high sensitivity have been widely used in deep-ultraviolet lithography for high-throughput applications. One of the most serious problems in chemically amplified resists is that their profile is degraded by alkaline contaminants such as amine and ammonia. Nitride-based BARC films that result in ammonia outgassing would cause the footing effect of chemically amplified resists during the postexposure bake (PEB) procedure. ${ }^{9}$ In this paper the silicon nitride film was found to have good thermal stability after an oxygen plasma treatment.

\section{Experimental Setup}

In our experiment, the silicon nitride film was deposited by employing a plasma-enhanced chemical vapor deposition system (Surface Technology System, Multiplex Cluster). The oxygen plasma treatment of BARC films was also carried out in this plasmaenhanced chemical vapor deposition system. The reflectance spectra were measured with a deepultraviolet and vacuum-ultraviolet optical spectrometer (Acton Research Corporation). The composition and depth profile of the optical-gradient film was measured by x-ray photoelectron spectroscopy (PHI15-255G) and secondary ion mass spectroscopy (CAMECA IMS-5F). The optical constants $n$ and $k$ are defined as the refractive index and the extinction coefficient in this paper. The optical constants of the films were obtained by the $R-T$ method with an N\&K analyzer. ${ }^{10,11}$ A thermal stability test was performed with a thermal desorption spectroscope (HITACHI, UG21) to observe whether there was base contamination from BARC films during the PEB procedure. The photoresist used for measuring swing effects was hydrogen silsesquioxane (HSQ), which has low absorption at $157 \mathrm{~nm} .{ }^{12}$ The conventional characteristic matrix model was used to simulate optical thin-film structures in this paper. ${ }^{13}$

\section{Results and Discussion}

The Si $2 p$ and N $1 s$ photoelectron spectra of the silicon nitride films after oxygen plasma treatment are shown in Figs. 2(a) and 2(b), respectively. The radio-frequency power during plasma treatment is

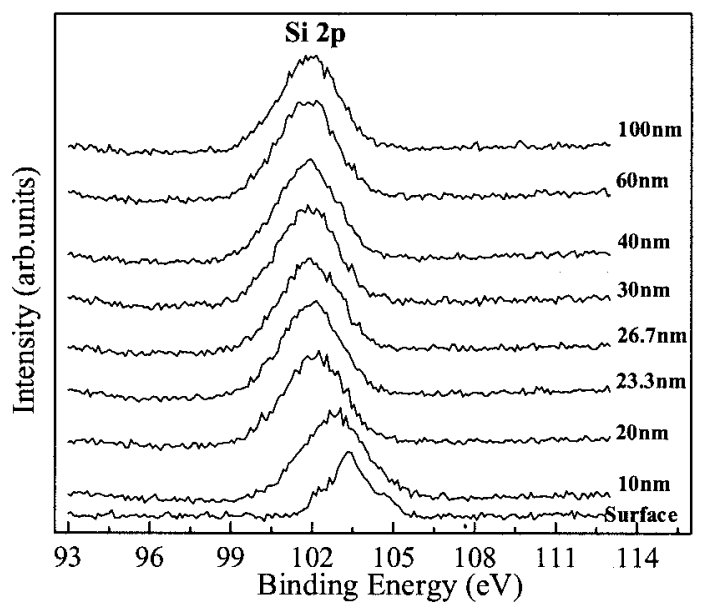

(a)

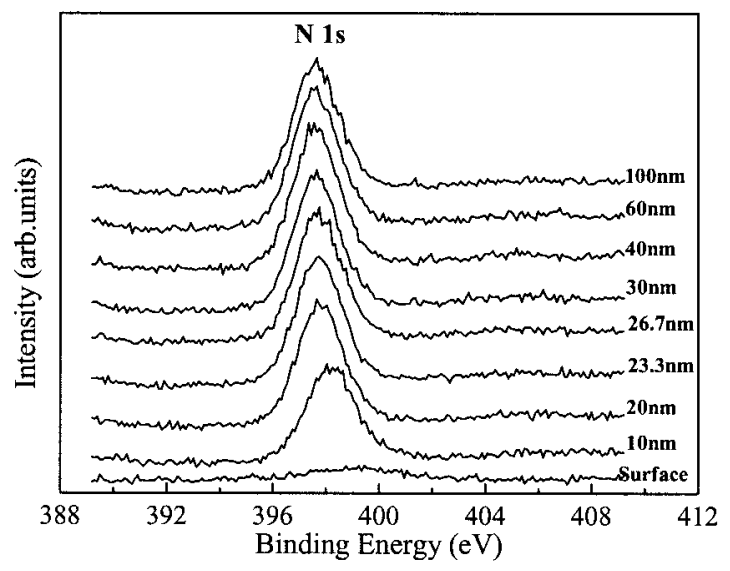

(b)

Fig. 2. (a) Si $2 p$ and (b) N $1 s$ photoelectron spectra of the silicon nitride film after oxygen plasma treatment.

$200 \mathrm{~W}$, and the time is $2 \mathrm{~min}$. The depth profile of the film is shown in Fig. 3. The Si $2 p$ peak gradually changes from $103.4 \mathrm{eV}$ to $101.8 \mathrm{eV}$ from the surface to the depth of $30 \mathrm{~nm}$ inside the oxygen-plasma-treated film, which indicates that the composition of the sur-

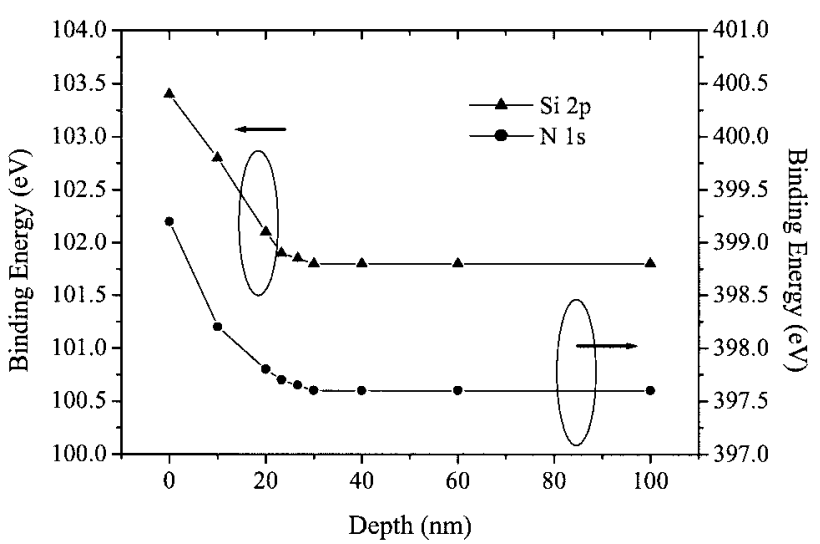

Fig. 3. Depth profile of the silicon nitride film after oxygen plasma treatment. 


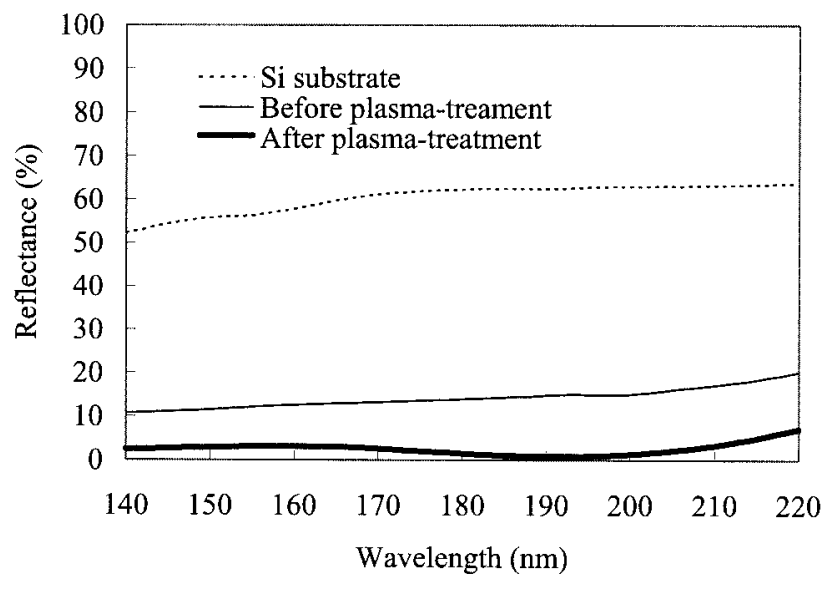

Fig. 4. Reflection spectra of a silicon substrate coated by a silicon nitride film before and after plasma treatment.

face of the silicon nitride film after oxygen plasma treatment is silicon dioxide $\left(\mathrm{SiO}_{2}, 103.4 \mathrm{eV}\right)$. The composition gradually changes from $\mathrm{SiO}_{2}$ to $\mathrm{Si}_{3} \mathrm{~N}_{4}$ $(101.8 \mathrm{eV})$ as the depth becomes greater than 30 $\mathrm{nm} .{ }^{14}$ Similarly, the $\mathrm{N} 1 s$ peak gradually changes from $399.2 \mathrm{eV}\left(\mathrm{NO}^{-}\right)$to $397.5 \mathrm{eV}\left(\mathrm{Si}_{3} \mathrm{~N}_{4}\right)$ from the surface to the depth of $30 \mathrm{~nm}$ inside the plasmatreated film. ${ }^{15}$ The intensity of the $\mathrm{N} 1 s$ peak at the surface is weak and then gradually increases inside the film. This indicates that the surface of the silicon nitride film is oxidized after oxygen plasma treatment, and the composition gradually changes to $\mathrm{Si}_{3} \mathrm{~N}_{4}$ as the depth becomes greater than $30 \mathrm{~nm}$. The compositional analysis of the optical-gradient film was carried out by using areas under the curves of Si $2 p, \mathrm{~N} 1 s$, and $\mathrm{O} 1 s$ with their respective sensitivity factors. ${ }^{14}$ The high oxygen composition of a film yields low absorption in the deep- and vacuumultraviolet regimes. The optical constants $(n, k)$ of the silicon nitride film after plasma treatment are also found to change gradually from $(1.684,0.052)$ to $(1.743,0.732)$ as simulated by the effective-medium model. Optical-gradient-types of antireflective coating therefore can be fabricated easily with the oxygen plasma treatment.

Figure 4 shows that the reflectance of a silicon substrate was measured as $\sim 58 \%$ and $\sim 62 \%$ at 157 and $193 \mathrm{~nm}$, respectively. The optical-gradient type of antireflective coating described above was used to reduce the reflectance of a silicon substrate. Adding

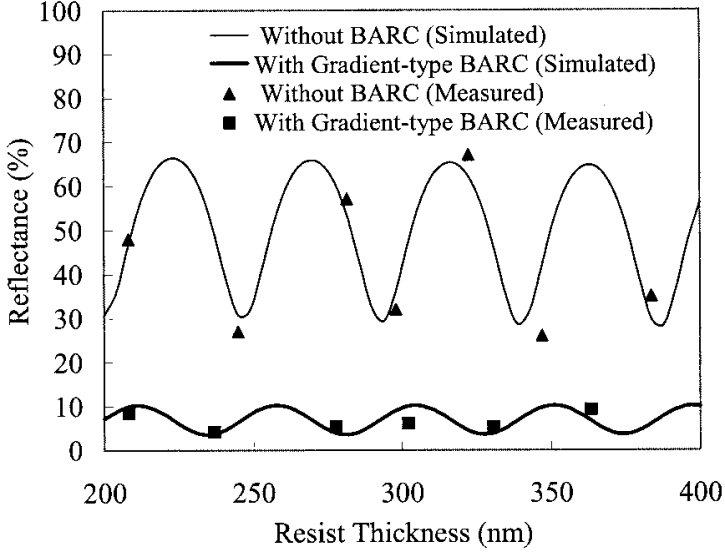

Fig. 5. Reflectance swing curves of resist coated on a silicon substrate with a gradient-type BARC structure at $157 \mathrm{~nm}$.

a single-layer silicon nitride film on a silicon substrate reduced the measured reflectance to $\sim 10 \%$. After a simple oxygen plasma treatment $(200 \mathrm{~W}, 2$ min), a reflectance of less than $2 \%$ can be achieved at both 193 and $157 \mathrm{~nm}$.

The optical-gradient type BARC was added to reduce the reflectance between the silicon crystal substrate and the resist interface. The thickness of the optical-gradient BARC film was $80 \mathrm{~nm}$. Based on thin-film theory, the simulated reflectance at $157 \mathrm{~nm}$ can be reduced to $0.53 \%$. The swing effect caused by optical interference between the fields reflected from the air-resist and resist-substrate interfaces will provide control of critical dimensions in an optical lithography process. The reflectance swing curves of the resist coated on a silicon wafer with and without a gradient-type BARC layer were simulated and measured, and the results are shown in Fig. 5. The reflectance exhibits a sinusoidal variation from $\sim 30 \%$ to $65 \%$ at $157 \mathrm{~nm}$ for a resist thickness range from 200 to $400 \mathrm{~nm}$ before the BARC layer is added and the variation is reduced to $\sim 4 \%$ to $10 \%$ after the addition. The results indicate that the gradient-type BARC can significantly reduce the swing effect arising from a resist. It is thus expected that using such a gradient-type BARC can improve the capability of controlling critical dimensions.

We discuss the practicality of using single-layer and gradient-type BARC structures for other highly reflective substrates such as polysilicon, tungsten,

Table 1. Single-Layer and Gradient-Type BARC Structures for Various Highly Reflective Substrates

\begin{tabular}{|c|c|c|c|c|c|c|c|}
\hline \multirow[b]{2}{*}{ Performance } & \multicolumn{7}{|c|}{ Substrate } \\
\hline & $\mathrm{Si}$ & Poly-Si & $\mathrm{W}$ & $\mathrm{Cu}$ & $\mathrm{TiN}$ & $\mathrm{TaN}$ & $\mathrm{Al}$ \\
\hline Optical constants at $157 \mathrm{~nm}$ & $(0.66,2.03)$ & $(0.89,2.08)$ & $(0.93,2.00)$ & $(1.03,1.03)$ & $(1.44,1.45)$ & $(1.53,0.97)$ & $(0.08,2.00)$ \\
\hline Reflectance from resist/substrate interface & $53.76 \%$ & $45.25 \%$ & $42.17 \%$ & $17.67 \%$ & $18.22 \%$ & $8.52 \%$ & $92.42 \%$ \\
\hline $\begin{array}{l}\text { Reflectance after adding a single-layer } \\
\text { BARC }\end{array}$ & $0.54 \%$ & $0.83 \%$ & $0.91 \%$ & $2.49 \%$ & $2.23 \%$ & $3.40 \%$ & $0.16 \%$ \\
\hline $\begin{array}{l}\text { Reflectance after adding a gradient-type } \\
\text { BARC }\end{array}$ & $0.53 \%$ & $0.55 \%$ & $0.56 \%$ & $0.65 \%$ & $0.62 \%$ & $0.67 \%$ & $0.48 \%$ \\
\hline
\end{tabular}




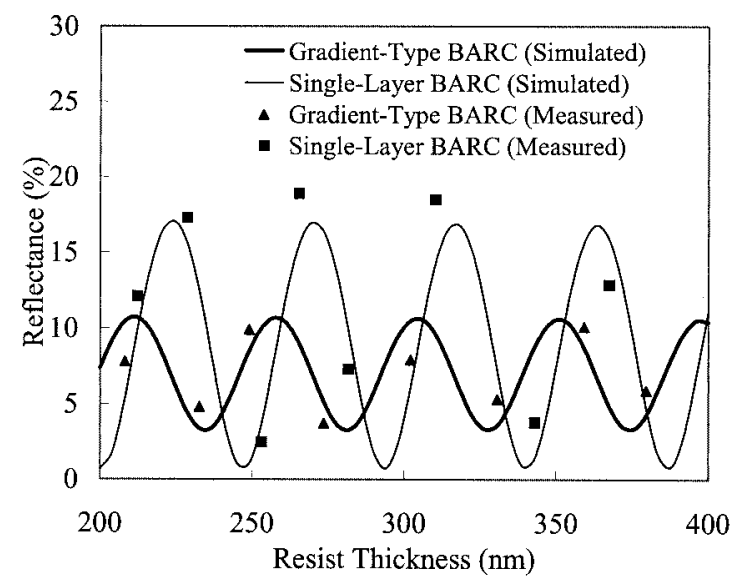

Fig. 6. Reflectance swing curves of resist coated on a tantalum nitride substrate with single-layer and gradient-type BARC structures at $157 \mathrm{~nm}$.

copper, titanium nitride, tantalum nitride, and aluminum, which are commonly used in semiconductor processes. These substrates are evaluated by simulating their optical performance summarized in Table 1. These results indicate that optical-gradient-type films could reduce the reflectance from the interface of the resist and highly reflective materials to less than $1 \%$ at $157 \mathrm{~nm}$, which is better than a singlelayer BARC. Figure 6 shows reflectance swing curves of single-layer and gradient-type BARC structures for tantalum nitride substrates at $157 \mathrm{~nm}$. Simulation and measured results indicate that the gradient-type BARC has a smaller swing ratio than the single-layer BARC. Similar results can also be obtained for $193 \mathrm{~nm}$. The optical-gradient film is therefore expected to have great potential as a BARC structure for various highly reflective substrates.

The thermal stability of silicon-nitride-based BARC layers was evaluated by thermal desorption spectroscopy. During the measurement the substrate is heated at a rate of $10{ }^{\circ} \mathrm{C} / \mathrm{min}$ to $400{ }^{\circ} \mathrm{C}$, which is far beyond the conventional PEB temperature $\left(\sim 100-150{ }^{\circ} \mathrm{C}\right)$ of resists. The spectrum for ammonia $(M / e=17)$ was measured with a quadrupole mass analyzer. A silicon dioxide film was used as an $\mathrm{NH}_{3}$ capping layer and was deposited over a silicon nitride film. Figure 7 shows the desorption curves for silicon nitride films covered by silicon dioxide films of different thicknesses. We found that the desorption curves are similar to the substrate background at an oxide film thickness of $75 \mathrm{~nm}$. For etching, the thickness of the BARC layer should be as small as possible. Another method is treating the silicon nitride surface with oxygen plasma. The plasma treatment method does not increase the thickness requirement of the BARC layer. The oxygen gas-flow rate is 100 standard $\mathrm{cm}^{3} / \min$ (sccm), the radio-frequency power is $200 \mathrm{~W}$, and the time period is varied from 1 to $10 \mathrm{~min}$. Figure 8 shows $\mathrm{NH}_{3}$ desorption curves indicating the dependence of the oxygen plasma treatment on time. When the

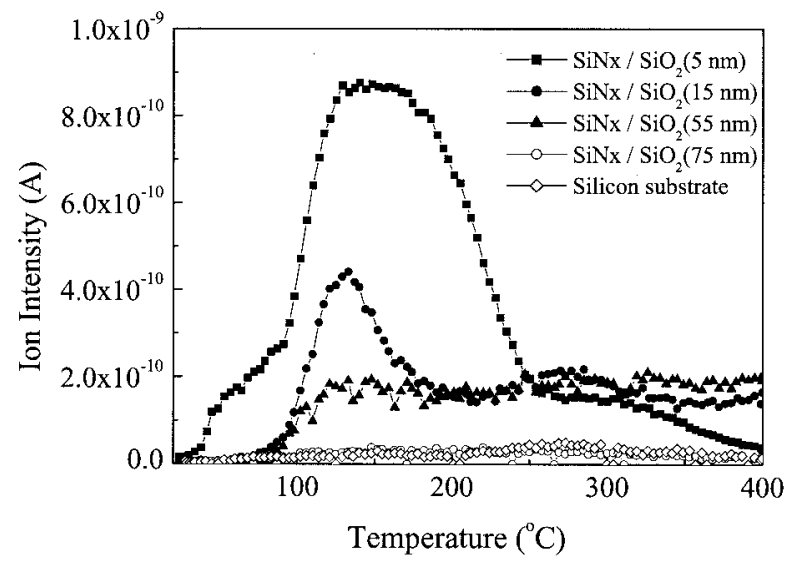

Fig. 7. $\mathrm{NH}_{3}$ desorption curves showing dependence of capped silicon dioxide films on thickness.

plasma treatment time increased, the $\mathrm{NH}_{3}$ desorption intensity first decreased rapidly and then saturated. This is because the surface of the silicon nitride film is oxidized after the plasma treatment. The surface oxide is preventing the $\mathrm{NH}_{3}$ desorption by the silicon nitride surface. Results indicate that the $\mathrm{NH}_{3}$ desorption curves are similar to those for the substrate background when the plasma treatment time exceeded $2 \mathrm{~min}$. Therefore this plasma treatment condition ( $200 \mathrm{~W}, 2 \mathrm{~min}$ ) was used to produce an optical-gradient BARC layer with good thermal stability during the PEB procedure as described above.

For a silicon-nitride-based single BARC layer structure with optical constants $(1.743,0.732)$ at 157 $\mathrm{nm}$ with a thickness of $20.8 \mathrm{~nm}$, the reflectance between the resist and the silicon-nitride-based BARC layer interface can be reduced to $0.54 \%$. The thickness tolerance analysis is shown in Fig. 9, which indicates that the reflectance will increase to $5 \%$ $20 \%$ when the thickness tolerance is larger than $60 \%$. Similarly, Fig. 9 also shows the thickness tolerance analysis for an optical-gradient BARC film with a thickness of $80 \mathrm{~nm}$ as described above. For the same $60 \%$ thickness tolerance, the reflectance can remain

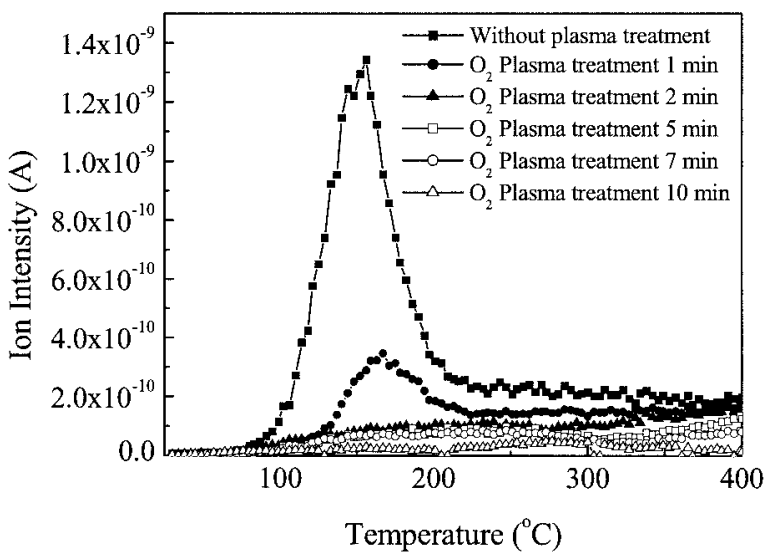

Fig. 8. $\mathrm{NH}_{3}$ desorption curves showing dependence of the oxygen plasma treatment procedure on time. 


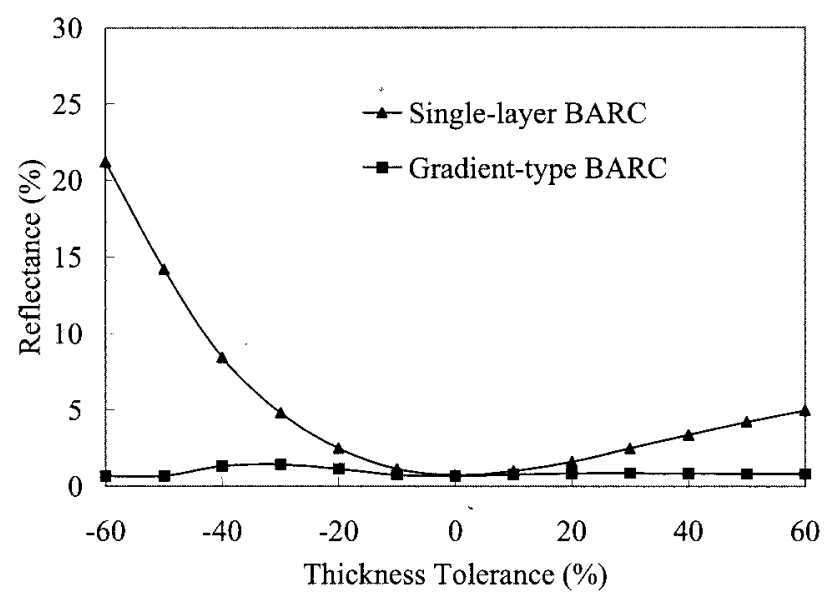

Fig. 9. Thickness tolerance analysis of a single-layer and an optical-gradient-type BARC structure.

less than $1.5 \%$, indicating that the optical-gradient structure can significantly increase the thicknesscontrolled tolerance.

\section{Conclusion}

In this paper we demonstrate an optical-gradient bottom antireflective coating film that can be easily prepared by treating a silicon nitride film with oxygen plasma. The surface of the silicon nitride film is oxidized after the plasma treatment. The oxygen composition is gradually decreased inside the silicon nitride film. After an oxygen plasma treatment procedure $(200 \mathrm{~W}, 2 \mathrm{~min})$, the oxidation depth is found to be $\sim 30 \mathrm{~nm}$. The optical constants $(n, k)$ are also found to change gradually from $(1.684,0.052)$ to $(1.743,0.732)$ at $157 \mathrm{~nm}$. A reflectance of less than $1 \%$ for various highly reflective substrates has been achieved. For $60 \%$ thickness tolerance, the reflectance can remain less than $1.5 \%$, indicating that the optical-gradient structure has a high thicknesscontrolled tolerance. The optical-gradient film is also shown to have high thermal stability during the postexposure bake procedure. Results indicate that the optical-gradient-type BARC is suitable in both $\mathrm{ArF}$ and $\mathrm{F}_{2}$ excimer lasers for sub-70-nm lithography applications.

The authors are very thankful to the National Science Council, Taiwan, Republic of China, for support in project NSC 92-2722-2-317-200.

\section{References}

1. Semiconductor Industry Association, International Technology Roadmap for Semiconductors 2001 ed. (Semiconductor for Industry Association, San Jose, Calif., 2001), http://public.itrs. net/Files/2001ITRS.

2. R. A. Cirelli, G. R. Weber, A. Kornblit, R. M. Baker, F. P. Klemens, and J. Demarco, "A multi-layer inorganic antireflective system for use in $248 \mathrm{~nm}$ deep ultraviolet lithography," J. Vac. Sci. Technol. B 14, 4229-4233 (1996).

3. L. A. Wang and H. L. Chen, "Multi-layer hexamethyldisiloxane film as bottom antireflective coating for ArF lithography," J. Vac. Sci. Technol. B 17, 2772-2775 (1999).

4. T. P. Ong and B. Roman, "CVD SiNx Anti-reflective coating for sub-0.5 micrometer lithography," in 1995 IEEE Symposium on VLSI Technology: Digest of Technical Papers (Institute of Electrical and Electronics Engineers, New York, 1995), p. 73.

5. Y. Trouiller, N. Buffet, T. Mourier, Y. Gobil, P. Schiavone, and

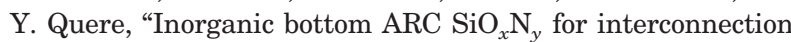
levels on 0.18- $\mu \mathrm{m}$ technology," in Advances in Resist Technology and Processing XV, W. Conley, ed., Proc. SPIE 3333, 324333 (1998).

6. C. H. Lin and L. A. Wang, "Feasibility of utilizing hexamethyldisiloxane film as a bottom antireflective coating for $157 \mathrm{~nm}$ lithography,” J. Vac. Sci. Technol. B 19, 2357-2361 (2001).

7. Y. Sato, Y. Onishi, Y. Nakano, and S. Hayase, "Spun-on carbon antireflective layer with etch resistance for deep and vacuum ultraviolet lithography processes," J. Vac. Sci. Technol. B 19, 2385-2388 (2001).

8. L. Ward, The Optical Constants of Bulk Materials and Films (Institute of Physics, Bristol, 1994), Chap. 8.

9. Y. Kawai, A. Tanaka, and T. Matsuda, "The effect of an organic base in chemically amplified resist on patterning characteristics using KrF lithography,” Jpn. J. Appl. Phys. 33, 7023-7027 (1994).

10. T. C. Paulick, "Inversion of normal-incidence $(R, T)$ measurements to obtain $n+i k$ for thin films," Appl. Opt. 25, 562 (1986).

11. A. R. Forouhi and I. Bloomer, "Optical properties of crystalline semiconductors and dielectrics,” Phys. Rev. B 38, 1865-1874 (1988).

12. V. Liberman, T. M. Bloomstein, and M. Rothschild, "Determination of optical properties of thin films and surfaces in 157-nm lithography," in Metrology, Inspection, and Process Control for Lithography XIV, N. T. Sullivan, ed., Proc. SPIE 3998, 480-490 (2000).

13. H. A. Macleod, Thin Film Optical Filters (Macmillan, New York, 1986), Chap. 2.

14. J. Chastain and R. C. King, Jr., Handbook of X-ray Photoelectron Spectroscopy (Physical Electronics, Perkin-Elmer, Eden Praire, Minn., 1995).

15. G. Latha, N. Rajendran, and S. Rajeswari, J. Mater. Eng. Perform. 6, 743-748 (1997). 